Gut, 1984, 25, 473-477

\title{
Oesophageal stricture and dysphagia after endoscopic sclerotherapy for bleeding varices
}

\author{
TIA SØRENSEN, F BURCHARTH, M L PEDERSEN, AND F FINDAHL \\ From the Department of Surgical Gastroenterology, Herlev Hospital, University of Copenhagen, \\ Copenhagen, Denmark
}

SUMMARY Oesophageal stricture and dysphagia after endoscopic sclerotherapy of oesophageal varices were assessed with regard to occurrence and severity and the relation to the treatment. We followed 34 patients for three to 47 months who had two to 25 treatments with submucosal, paravenous injections of polidocanol $(3 \%)$. Twenty patients $(59 \%)$ developed stricture or dysphagia; 14 both dysphagia and endoscopically verified stricture, two dysphagia without stricture, and four stricture without dysphagia. Both phenomena occurred intermittently and often independent of each other, but occupied median $38 \%$ of the observation time in these 20 patients. The patients developing strictures had received significantly more treatments and greater amount of sclerosant, and they had significantly more preceding mucosal necroses. The varices were eradicated to about the same degree and the incidence of recurrent haemorrhage was the same as in the patients who had not developed stricture.

Endoscopic sclerotherapy apparently improves the control of haemorrhage from oesophageal varices and prolongs survival. ${ }^{12}$ Several acute complications have been encountered: oesophagitis, necroses, ulcerations, tears, perforation, fistula formation, and mediastinitis, some of which, although rarely, have been fatal. ${ }^{1-8}$ The inflammation and necroses followed by fibrosis might result in motility disturbances, ${ }^{8-10}$ stricture and dysphagia. In clinical studies, however, these complications have been reported only occasionally. We investigated the occurrence of oesophageal stricture and dysphagia, their antecedents and the relation to the intensity and the effects of the treatment.

\section{Methods}

PATIENTS

Fifty seven patients were admitted with haemorrhage from oesophageal varices and endoscopic sclerotherapy was started in all cases irrespective of the clinical assessment of liver function. The age range was $15-82$ years with a median of 52 years. During the first three months

Address for correspondence: F Burcharth, dr med, Dept of Surgical Gastroenterology D 106, Herlev Hospital, DK-2730 Herlev, Denmark.

Received for publication 29 July 1983 after diagnosis of varices, 23 patients $(40.4 \%)$ died, which mortality compares well with the natural history of this disease. ${ }^{11}$ As the oesophageal stricture and dysphagia are of clinical interest only for patients escaping the initial hazards of the disease, we selected for this report the 34 patients who survived three months or more. They were observed a median of 28 (range 3-47) months. Twenty five had alcoholic cirrhosis, six had nonalcoholic cirrhosis, and three had portal vein thrombosis. When the sclerotherapy began 14 had ascites and seven were in hepatic precoma, none in coma.

\section{SCLEROTHERAPY}

Sclerotherapy was performed with a straight viewing fibre-optic endoscope (Olympus GIF-K or TGF-2D) and a $4 \mathrm{~mm}$ needle (Olympus NM3) under sedation with diazepam (Stesolid) 5-15 $\mathrm{mg}$ and fentanyl (Haldid) $0 \cdot 1-0 \cdot 2 \mathrm{mg}$. The sclerosant was polidocanol (Aethoxysklerol) $30 \mathrm{mg} / \mathrm{ml}$ of water with $0.05 \mathrm{ml}$ of ethanol. During each treatment session $0.5-1.0 \mathrm{ml}$ was injected in the submucosa at 10-15 sites along each variceal column from the gastro-oesophageal junction and proximally with intervals of $1-3 \mathrm{~cm}$. It was planned to place the sclerosant as a papule in the paravariceal tissue although occasional intravariceal injection was unavoidable as judged from the lack of a papule and 
the bleeding after withdrawal of the needle. The rationale of the proximal extension of the sclerosing is that a few patients have been seen bleeding from proximal varices after sclerosing of the varices in the distal $5 \mathrm{~cm}$ where rupture frequently occurs in untreated patients. Usually, the first treatment was given as soon as the patient was resuscitated and haemostasis was obtained with the SengstakenBlakemore tube. The treatment was repeated every third day until the varices disappeared or did not bleed when punctured. If ulcers or necroses appeared, treatment was interrupted while the lesions healed. After discharge the patients were followed by endoscopy with increasing intervals. If varices appeared, a new series of treatments was undertaken.

\section{OBSERVATIONS}

At each endoscopy during the observation period the following variables were recorded: degree of oesophageal varices (scale $0,1,2,3$ ); dysphagia (scale 0,1 ) for solid or liquid food; presence of stricture, whether or not it was passable for the endoscope, stricture site measured from the teeth to the upper limit; presence of mucosal necroses (scale 0,1 ); oesophageal ulceration (scale 0,1 ).

\section{ANALYSES}

We investigated the relationship between occurrence of oesophageal stricture and the intensity of treatment (number of treatment sessions, amount of sclerosant given per session and cumulated over time) effects of the treatment (variceal eradication, incidence of recurrent haemorrhage, observation time without rebleeding) and antecedents of stricture (preceding dysphagia, mucosal necroses, and ulceration). As the strictures developed after different time intervals, it was necessary for this investigation to define a control value for each patient developing a stricture. This control value should express the observations in those patients who were at risk of developing, but did not develop a stricture during the same observation period. Thus, for the number of treatment sessions, as an example, the number given until the stricture was diagnosed in one patient was compared with a matched control value calculated as the average number of treatments given to those who were alive and had not, or not yet, developed a stricture during the same period of observation. The number of patients from whom the control values were derived declined from 33 for the earliest diagnosed stricture to five for the latest diagnosed.

Statistical comparisons of the stricture patients with their corresponding control values were carried out with the Wilcoxon's matched-pairs test.

\section{Results}

During the entire course of observation 20 of the 34 patients developed stricture and/or dysphagia; 14 stricture and dysphagia, two dysphagia without stricture, and four stricture without dysphagia. In the 20 patients, stricture and/or dysphagia were present during median 38\% (range 1-86\%) of their observation time (Fig. 1). Both phenomena occurred intermittently and often independently of each other (Fig. 1).

The time of first diagnosis of the stricture ranged from nine to 1229 days with a median of 206 days after start of sclerotherapy. At the first appearance of the stricture, eight out of the 18 patients presented a stricture not passable for the endoscope. Except in four patients all strictures were located to the lower $5 \mathrm{~cm}$ of the oesophagus. Ten of the patients complained of dysphagia at the time of stricture diagnosis.

The occurrence of oesophageal complications within each of the four years of observation may be assessed as the number of patients affected in per cent of the mean number of patients under observation during each year (Table). About half of the patients suffered from dysphagia and/or stricture each year. Dysphagia for solid food occurred in about one third, whereas dysphagia for liquid food was rare, not exceeding 10 per cent. A stricture passable for the endoscope was found in about $20 \%$ each year. The frequency of stricture not passable increased from $13 \%$ to $33 \%$ during the four years. The correlation between occurrence of dysphagia and stricture was only moderate (Table).

Endoscopic oesophageal dilatation (the EderPuestow technique to olive size no 45) was performed in 11 patients, two of whom had dysphagia without stricture (Fig. 1). One patient developed a narrow stricture $5 \mathrm{~cm}$ above the cardia 14 months after start of treatment. Dilatation led to a transdiaphragmatic fistula. Surgical correction with fundoplication was followed by narrowing of the stricture necessitating 10 dilatations during 18 months.

The patients developing a stricture had received median eight treatments with a total of median 123 $\mathrm{ml}$ sclerosant (Fig. 2). The median of the control values were 7.4 treatments and $93.0 \mathrm{ml}$ sclerosant, and the differences were significant $(p<0 \cdot 01)$ (Fig. 2 ). Until stricture was diagnosed, the amount of sclerosant given at each session was median $14.3 \mathrm{ml}$ (range 11.9-23.3 ml) which was significantly more than the control value having a median of $12.7 \mathrm{ml}$ (range $10 \cdot 9-15 \cdot 1 \mathrm{ml})(\mathrm{p}<0 \cdot 01)$.

The effect of sclerotherapy on eradication of the oesophageal varices was not significantly related to 

patient subjected to sclerotherapy for oesophageal varices: occurrence of dysphagia, oesophageal stricture, and dilatation in relation to time of observation.
Fig. 1 Clinical course for each

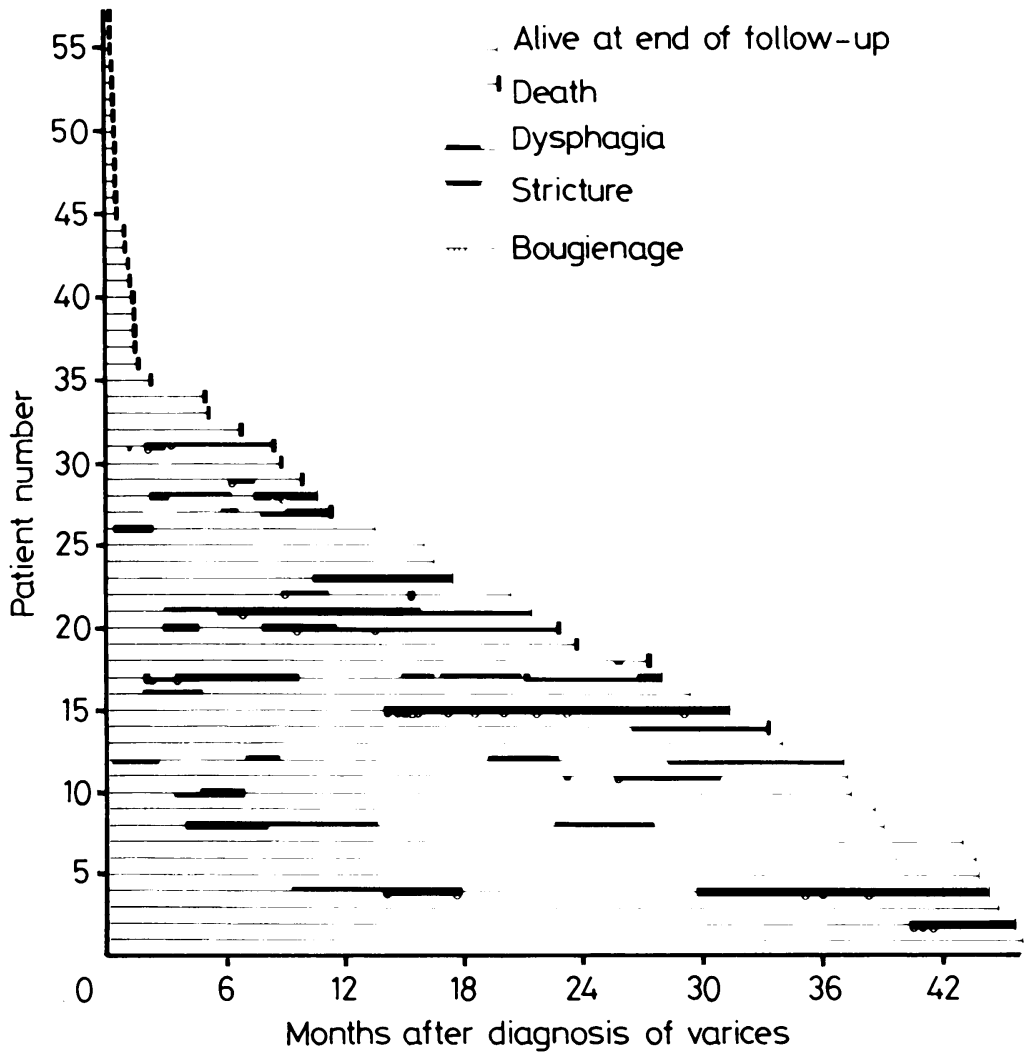

the development of oesophageal stricture. Before sclerotherapy the median size of the varices was 2.33 (range two to three) among the patients developing strictures, and the median control value was $2 \cdot 08$. At the time the strictures were diagnosed, the median size of the varices had decreased to 0.53 among stricture patients to be compared with a median control value of $0 \cdot 80$. Eleven of the patients developing strictures had at least one episode of recurrent bleeding which did not differ from the

Table Dysphagia and stricture during each year after start of sclerotherapy for bleeding oesophageal varices.

\begin{tabular}{|c|c|c|c|c|c|c|c|c|}
\hline & \multicolumn{8}{|c|}{ Year after start of therapy } \\
\hline & \multicolumn{2}{|l|}{ lst } & \multicolumn{2}{|c|}{$2 n d$} & \multicolumn{2}{|c|}{$3 r d$} & \multicolumn{2}{|c|}{$4 t h$} \\
\hline & $n$ & $\%^{*}$ & $n$ & $\% *$ & $n$ & $\% *$ & $n$ & $\%^{*}$ \\
\hline Number at start of the year & 34 & & 26 & & 18 & & 12 & \\
\hline Average number observed & 30 & 100 & 22 & 100 & 16 & 100 & 6 & 100 \\
\hline \multicolumn{9}{|l|}{ Dysphagia } \\
\hline For solid food & 11 & 37 & 7 & 32 & 4 & 25 & 2 & 33 \\
\hline For liquid food & 3 & 10 & 1 & 5 & 0 & 0 & 0 & 0 \\
\hline \multicolumn{9}{|l|}{ Stricture } \\
\hline Passable for the endoscope & 6 & 20 & 5 & 23 & 3 & 19 & 1 & 17 \\
\hline Not passable & 4 & 13 & 3 & 14 & 4 & 25 & 2 & 33 \\
\hline \multicolumn{9}{|l|}{ Combinations } \\
\hline Dysphagia and stricture & 9 & 30 & 6 & 27 & 3 & 19 & 2 & 33 \\
\hline Dysphagia without stricture & 5 & 17 & 2 & 9 & 1 & 6 & 0 & 0 \\
\hline Stricture without dysphagia & 1 & 3 & 2 & 9 & 4 & 25 & 1 & 17 \\
\hline
\end{tabular}

* Per cent of average number of patients observed during the year. 

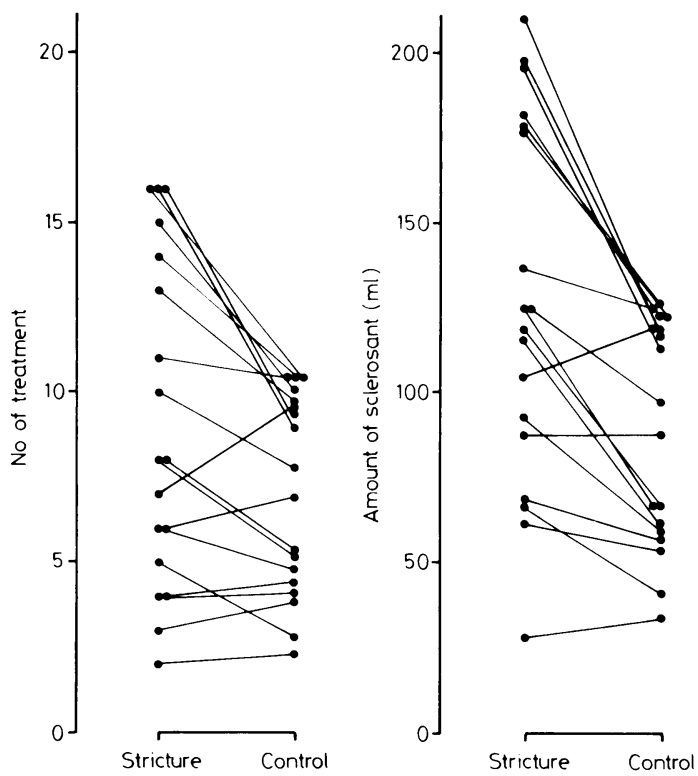

Fig. 2 Number of treatments and amount of sclerosant received by patients developing strictures compared with control values estimated from patients who at time had not developed stricture.

control value of $11 \cdot 2$. The observation time since start of therapy without recurrent bleeding was median 278 days (range 21-1343 days) among stricture patients which did not differ significantly from the control values with a median of 503 days.

Four patients complained of dysphagia before the stricture was diagnosed; this was higher, but not significantly different from the control value of 1.69 . Mucosal necroses were seen in 15 patients before stricture developed; the control value was $10 \cdot 6$ and significantly lower $(p<0 \cdot() 2)$. Five patients had oesophageal ulcers before the stricture, and the control value was $2 \cdot 67$, but this difference was not significant.

\section{Discussion}

In this study stricture and/or dysphagia occurred in about half of the patients during the four years observation period after start of sclerotherapy. This is a higher incidence than reported in other studies. In a recent prospective trial including 51 patients assigned to sclerotherapy nine patients $(18 \%)$ developed stricture, five of whom complained of dysphagia. ${ }^{2}$ An even lower incidence has been reported in the other studies. ${ }^{3-7}$ Our study is not directly comparable with these studies, however, as none of them has taken into account the time of occurrence, and the duration of the complications in relation to the changing number of patients under observation. On the other hand, the treatment was carried out more aggressively here than in most other studies. We injected sclerosant every third day against - for example, three to four weeks interval in the prospective trial. ${ }^{2}$ Also the extension of the treatment up to about $10 \mathrm{~cm}$ proximal to the gastro-oesophageal junction. to which the treatment usually is restricted, may have contributed to the high incidence of dysphagia and stricture. The choice of the aggressive treatment regimen was made with the idea of offering the possible benefits of this treatment to the patients who are subject to the high initial mortality after the first episode of haemorrhage from varices. ${ }^{11}$

The oesophageal complications occurred intermittently and were present in median $38 \%$ of the total observation time. but with a large inter- and intrapatient variation. The severity of the complications is shown by the findings of some patients complaining of dysphagia for liquids, of a frequency of narrow strictures increasing from 13\% through $33 \%$, and the necessity of dilatation in 11 patients.

Dysphagia was not always ascribable to a detectable stricture and several patients had strictures not resulting in dysphagia. The inflammation and ulceration of the oesophageal mucosa may cause motility disturbances with dysphagia. ${ }^{9}$ 11" On the other hand, presence of a stricture without dysphagia might reflect an uncertain interpretation of the endoscopic findings as well as a variable subjective threshold for dysphagia.

The sclerotherapy has many variables of possible influence on the development of dysphagia and stricture: intra- $v s$ paravariceal injection, amount, concentration and type of sclerosant, the vehicle of the sclerosant, the number of injections per treatment, depth of and distance between injections, total number and time interval between the treatments, criteria for interruption and cessation of treatment. We examined the relation to the cumulated number of treatments and amounts of sclerosant. The stricture patients had most treatments with greatest amount of sclerosant, but this did not distinguish them clearly from those without stricture.

It is plausible that less aggressive treatment might prevent stricture formation, but this will probably be at the expense of an increased risk of recurrent variceal bleeding. In our study, the patients developing strictures showed tendencies to greater varices at start of treatment, slightly more effective eradication of varices and a shorter observation time without recurrent bleeding. Although these differ- 
ences were statistically insignificant, they suggest that the more aggressive sclerotherapy was administered on the basis of a judgment of a greater need of therapy. A confident evaluation of these problems, however, require clinical controlled trials.

Several authors have discussed whether injection of the sclerosant should be intra- or paravariceal. ${ }^{4-6}$ It is often difficult to observe where the sclerosant actually is injected, and a planned intravariceal injection may, now and then, become paravariceal and vice versa. Stenosis and dysphagia is experienced with both kinds of intended injection sites. A comparative study with randomisation is required for a valid choice between them.

In order to identify the patients later developing strictures we assessed the occurrence of preceding dysphagia, mucosal necroses, and ulceration. Necroses occurred significantly more frequently in patients developing stenoses, but the frequency in the control series was also rather high. Preceding dysphagia and oesophageal ulceration were rare and not significantly more frequent than in the control series.

We conclude that stricture and dysphagia constitute a continuing and prominent clinical problem among the patients with oesophageal varices treated by sclerosing injections. The complications are more frequent in the most intensively treated patients, but it is questionable whether the treatment can be moderated without increased risk of variceal bleeding. Although the complications can be severe and require dilatation, we think that the beneficial effects of sclerotherapy ${ }^{12}$ outweigh these complications.

\section{References}

1 Clark AW, Westaby D, Silk DBA et al. Prospective controlled trial of injection sclerotherapy in patients with cirrhosis and recent variceal haemorrhage. Lancet 1980; 2: 552-4.

2 MacDougall BRD, Westaby D, Theodossi A, Dawson $J L$, Williams $R$. Increased long-term survival in variceal haemorrhage using injection sclerotherapy. Lancet 1982; 1: 124-7.

3 Johnston GW, Rodgers HW. A review of 15 years experience in the use of sclerotherapy in the control of acute haemorrhage from oesophageal varices. $\mathrm{Br} J$ Surg 1973; 60: 797-800.

4 Paquet KJ, Oberhammer E. Sclerotherapy of bleeding oesophageal varices by means of endoscopy. Endoscopy 1978; 10: 7-12.

5 Sinnet HD, Murray-Lyon IM, Reynolds KW, Johnson AG. Long-term injection sclerotherapy of esophageal varices. Gastroenterology 1979; 76: 1250.

6 Terblanche J, Northover JMA, Bornman P et al. A prospective controlled trial of sclerotherapy in the long-term management of patients after oesophageal varices bleeding. Surg Gynecol Obstet 1979; 148: 323-33.

7 Barsoum MS, Mooro HAW, Bolous FI, Ramzy AF, Rizk-Allah MA, Mahmoud FI. The complications of injection sclerotherapy of bleeding oesophageal varices. Br J Surg 1982; 69: 79-81.

8 Evans DMD, Jones DB, Cleary BK, Smith PM. Oesophageal varices treated by sclerotherapy: a histopathological study. Gut 1982; 23: 615-20.

9 Ogle SJ, Kirk CJ, Bailey RJ, Johnson AG, Williams R, Myrray-Lyon IM. Esophageal function in cirrhotic patients undergoing injection sclerotherapy for esophageal varices. Digestion 1978; 18: 178-85.

10 Sauerbruch T, Wirsching R, Leisner B, Weinzierl M, Pfahler M, Paumgartner G. Esophageal function after sclerotherapy of bleeding varices. Scand J Gastroenterol 1982; 17: 745-51.

11 Garceau AJ, Chalmers TC. The Boston Inter-Hospital Liver Group. The natural history of cirrhosis - survival with esophageal varices. $N$ Engl J Med 1963; 268: 469-73. 